\title{
ON MAPPING INDECOMPOSABLE CONTINUA ONTO CERTAIN CHAINABLE INDECOMPOSABLE CONTINUA
}

\author{
J. W. ROGERS, JR.
}

1. Introduction. The classical results on mappings (i.e. continuous transformations) involve arcs, either as the domain space (the HahnMazurkiewicz theorem) or as the range space (Urysohn's characterization of normality). Not enough of whatever common structure exists among more complicated continua (compact connected metric spaces) has as yet been perceived to allow the formulation of many mapping theorems of a general nature. Among the known results is the fact that not every continuum is a continuous image of a plane continuum [5], while every continuum is an image of some one-dimensional continuum [8], [11] (and hence of a continuum in $E^{3}$ ). Studies of circle-like continua have also yielded interesting theorems, [3], [13].

Even indecomposable continua, generally considered highly pathological, have shown promise of having interesting mapping properties. The example of a chainable continuum which can be mapped onto every chainable continuum, given in [4], [9], and [12], in each case was the pseudo-arc, which is hereditarily indecomposable. A continuum with similar properties with respect to circularly chainable continua has been found, [13], and again is hereditarily indecomposable.

In this paper, we show in $\$ 2$ that there is a chainable indecomposable continuum $D$ which is a continuous image of every indecomposable continuum. In $\S 3$, we study some indecomposable continuous images of $D$ (each of which is also, of course, an image of every indecomposaable continuum).

It is hoped the techniques used here will prove useful in further studies of the mapping properties of indecomposable continua; for example, to settle the following questions.

(1) Is the pseudo-arc a continuous image of every hereditarily indecomposable continuum?

(2) Is every continuum a continuous image of some (hereditarily) indecomposable continuum?

The author is grateful for the referees' suggestions, which led to several substantial improvements in this paper. 1969.

Presented to the Society, April 19, 1969; received by the editors February 28, 


\section{Mapping indecomposable continua onto $D$.}

Definitions. By a map we mean a continuous transformation. A polyhedron is a nondegenerate finitely triangulable continuum. By an inverse sequence $\left\{P_{i}, f_{i}\right\}$ on polyhedra we mean a sequence such that for each positive integer $i, P_{i}$ is a polyhedron and $f_{i}$ maps $P_{i+1}$ onto $P_{i}$. We use $\pi_{n}$ to denote the projection map from $\lim \left\{P_{i}, f_{i}\right\}$ onto $P_{n}$. If $i<j, f_{i j}=f_{i} \cdots f_{j-1}$. Let $I=[0,1]$.

Let $D=\lim \left\{I, g_{i}\right\}$, where for each $i, g_{i}(x)=2 x$ if $0 \leqq x \leqq \frac{1}{2}$, and $g_{i}(x)=2-2 x$ if $\frac{1}{2} \leqq x \leqq 1 . D$ is homeomorphic to a standard example of an indecomposable plane continuum, described in [1, Example 6, p. 662]. The next theorems show that any indecomposable continuum can be mapped onto $D$.

It is known that every continuum is the limit of an inverse sequence on polyhedra [6] (the result may also be obtained from [10]).

We suppose in Theorems $1-4$ that $M$ is an indecomposable continuum which is the limit of an inverse sequence $\left\{P_{i}, f_{i}\right\}$ on polyhedra.

Theorem 1. If $\delta>0, n$ is a positive integer, and $A$ and $B$ are points of $P_{n}$, then there are a positive integer $m>n$ and points $A^{\prime}$ and $B^{\prime}$ of $P_{m}$ such that $d\left[A, f_{n m}\left(A^{\prime}\right)\right]<\delta, d\left[A, f_{n m}\left(B^{\prime}\right)\right]<\delta$, and if $\alpha$ is an arc in $P_{m}$ from $A^{\prime}$ to $B^{\prime}$, then $d\left[B, f_{n m}(\alpha)\right]<\delta$.

Proof. Suppose not. Then there are a number $\epsilon>0$, a positive integer $n$, and points $A$ and $B$ of $P_{n}$ such that if $m>n$ and $A^{\prime}$ and $B^{\prime}$ are points of $P_{m}$ such that $d\left[A, f_{n m}\left(A^{\prime}\right)\right]<\delta$ and $d\left[A, f_{n m}\left(B^{\prime}\right)\right]<\delta$, then there is an $\operatorname{arc} \alpha$ in $P_{m}$ from $A^{\prime}$ to $B^{\prime}$ such that $d\left[B, f_{n m}(\alpha)\right] \geqq \delta$. Let $X$ and $Y$ denote points in different composants of $M$ such that $d\left[A, \pi_{n}(X)\right]<\delta$ and $d\left[A, \pi_{n}(Y)\right]<\delta$. Then there are an increasing sequence of positive integers $\left\{n_{i}\right\}$ and a sequence of arcs $\left\{\alpha_{i}\right\}$ such that for each $i, \alpha_{i}$ is an arc in $P_{n_{i}}$ from $\pi_{n_{i}}(X)$ to $\pi_{n_{i}}(Y)$ and $d[B$, $\left.f_{n n_{i}}\left(\alpha_{i}\right)\right] \geqq \delta$. The limiting set $L$ of the sequence $\pi_{n_{1}}^{-1}\left(\alpha_{1}\right), \pi_{n_{2}}^{-1}\left(\alpha_{2}\right), \cdots$ of subsets of $M$ is a subcontinuum of $M$ that contains both $X$ and $Y$, but no point of $\pi_{n}^{-1}(B)$, contrary to the fact that $M$ is irreducible from $X$ to $Y$.

Theorem 2. If $\epsilon>0, n$ is a positive integer, and $A$ and $B$ are points of $P_{n}$, then there are (1) an integer $m>n,(2)$ points $A^{\prime}$ and $B^{\prime}$ of $P_{m}$, and (3) a map $\phi$ from $P_{n}$ onto $P_{n}$ such that

(a) $d(\phi, 1)<\epsilon$ (where 1 denotes the identity on $\left.P_{n}\right)$,

(b) $A=\phi f_{n m}\left(A^{\prime}\right)=\phi f_{n m}\left(B^{\prime}\right)$, and

(c) $\left[\phi f_{n m}\right]^{-1}(B)$ separates $A^{\prime}$ from $B^{\prime}$ in $P_{m}$. 
Proof. There exist (1) connected open sets $R_{A}$ and $R_{A}^{\prime}$, containing $A$, of diameter $<\epsilon$, such that $\bar{R}_{A}^{\prime}$ lies in $R_{A}$, (2) similar sets $R_{B}$ and $R_{B}^{\prime}$ for $B$, and (3) a map $\phi$ from $P_{n}$ onto $P_{n}$ such that

(a) $\bar{R}_{A}$ and $\bar{R}_{B}$ are mutually exclusive,

(b) $\phi$ is the identity on $P_{n}-\left(R_{A}+R_{B}\right)$,

(c) $\phi\left(R_{A}^{\prime}\right)=A$ and $\phi\left(R_{B}^{\prime}\right)=B$, and

(d) $\phi\left(R_{A}\right)=R_{A}$ and $\phi\left(R_{B}\right)=R_{B}$. Clearly $d(\phi, 1)<\epsilon$.

Let $\delta$ denote a positive number less than $d\left(A, P_{n}-R_{A}^{\prime}\right)$ and $d\left(B, P_{n}-R_{B}^{\prime}\right)$, and let $m, A^{\prime}$, and $B^{\prime}$ be as given in Theorem 1 with respect to $\delta, n, A$, and $B$. Since, from Theorem $1, d\left[A, f_{n m}\left(A^{\prime}\right)\right]<\delta$, $f_{n m}\left(A^{\prime}\right)$ lies in $R_{A}^{\prime}$, so $\phi f_{n m}\left(A^{\prime}\right)=A$. Similarly, $\phi f_{n m}\left(B^{\prime}\right)=A$. Now, if $\alpha$ is an arc in $P_{m}$ from $A$ to $B$, then there is a point $Y$ of $\alpha$ such that $d\left[B, f_{n m}(Y)\right]<\delta$, and so $\phi f_{n m}(Y)=B$. Thus, since every arc in $P_{m}$ from $A^{\prime}$ to $B^{\prime}$ intersects $\left[\phi f_{n m}\right]^{-1}(B)$, that set separates $A^{\prime}$ from $B^{\prime}$ in $P_{m}$.

TheOREм 3. There are (1) an inverse sequence $\left\{P_{i}^{\prime}, g_{i}\right\}$ on polyhedra and (2) sequences $\left\{A_{i}\right\}$ and $\left\{B_{i}\right\}$ such that for each $i$,

(a) $A_{i}$ and $B_{i}$ are points of $P_{i}$,

(b) $A_{i}=g_{i}\left(A_{i+1}\right)=g_{i}\left(B_{i+1}\right)$,

(c) $g_{i}^{-1}\left(B_{i}\right)$ separates $A_{i+1}$ from $B_{i+1}$ in $P_{i+1}$, and

(d) there is a map that throws $M$ onto $\lim \left\{P_{i}, g_{i}\right\}$.

Proof. We define $\left\{P_{i}^{\prime}, g_{i}\right\}$ inductively from the sequence $\left\{P_{i}, f_{i}\right\}$ for $M$. Let $P_{1}^{\prime}=P_{1}$, and let $A_{1}$ and $B_{1}$ denote points of $P_{1}^{\prime}$. For each $n$, let $b_{n}=2^{-n}$ and let $a_{1}=m_{1}=1$. Let $m_{2}, A_{2}, B_{2}$, and $\phi_{1}$ satisfy the conditions of Theorem 4 for $m, A^{\prime}, B^{\prime}$, and $\phi$, respectively, regarding both $\epsilon$ and $n$ in that theorem as 1 , and $A$ and $B$ as $A_{1}$ and $B_{1}$, respectively. Let $g_{1}=\phi_{1} f_{m_{1} m_{2}}$ and $P_{2}^{\prime}=P_{m_{2}}$.

In general, given, for $j>1, g_{j-1}, m_{j}, P_{j}^{\prime}=P_{m_{j}}, A_{j}$, and $B_{j}$, let $a_{j}$ denote a positive number such that if $i<j$ and $X$ and $Y$ are points of $P_{j}^{\prime}$, and $d(X, Y)<a_{j}$, then $d\left[g_{i j}(X), g_{i j}(Y)\right]<b_{j}$. Let $m_{j+1}, A_{j+1}$, $B_{j+1}$, and $\phi_{j}$ satisfy the conditions of Theorem 2 for $m, A^{\prime}, B^{\prime}$, and $\phi$, respectively, regarding $\epsilon$ as $a_{j}, n$ as $m_{j}, A$ as $A_{j}$ and $B$ as $B_{j}$. Let $g_{j}=\phi_{j} f_{m_{j} m_{j+1}}$ and $P_{j+1}^{\prime}=P_{m_{j+1}}$.

Then $\left\{P_{i}^{\prime}, g_{i}\right\}$ is an inverse sequence, and the sequences $\left\{A_{i}\right\}$ and $\left\{B_{i}\right\}$ satisfy requirements (a)-(c) of this theorem. For each $i$, let $f_{i}^{\prime}=f_{m_{i} m_{i+1}}$. Then for each $i, d\left(f_{i}^{\prime}, g_{i}\right)<a_{i}$. Hence if (1) $S=\lim \left\{P_{i}^{\prime}, f_{i}^{\prime}\right\}$, which is homeomorphic to $M$, and (2) $T=\lim \left\{P_{i}^{\prime}, g_{i}\right\}$, then the conditions of M. Brown's Theorem 1 [2, p. 479] are satisfied, for the sequence $\left\{a_{i}\right\}$ defined above is a Lebesgue sequence for $\left\{P_{i}^{\prime}, g_{i}\right\}$, in Brown's terminology. (The reader should note that Brown's notation for inverse limits differs from the author's. In particular Brown's re- 
quirement " $\left\|f_{i+1}-g_{i+1}\right\|<a_{i}$ " in the notation of this paper would be " $\left\|f_{i}-g_{i}\right\|<a_{i}$ ".) Hence there is a map from $S$ onto $T$, and so $M$ can be mapped onto $T$.

Theorem 4. $M$ can be mapped onto $D$. Hence $D$ is a chainable indecomposable continuum which is a continuous image of every indecomposable continuum.

Proof. Let $\left\{P_{i}^{\prime}, g_{i}\right\},\left\{A_{i}\right\}$, and $\left\{B_{i}\right\}$ denote sequences as defined in Theorem 3 such that $M$ can be mapped onto $\lim \left\{P_{i}^{\prime}, g_{i}\right\}$. We show that $\lim \left\{P_{i}^{\prime}, g_{i}\right\}$ can be mapped onto $D$.

For each $n$, let $\alpha_{n+1}$ and $\beta_{n+1}$ denote two mutually separated subsets of $P_{n+1}$ such that $A_{n+1}$ lies in $\alpha_{n+1}, B_{n+1}$ lies in $\beta_{n+1}$, and $\alpha_{n+1}+\beta_{n+1}$ $=P_{n+1}^{\prime}-g_{n}^{-1}\left(B_{n}\right)$. Let $\left\{\phi_{i}\right\}$ denote a sequence of maps such that (1) $\phi_{1}$ maps $P_{1}^{\prime}$ onto $[0,1]$ and $\phi_{1}\left(A_{1}\right)=0$ and $\phi_{1}\left(B_{1}\right)=1$, and (2) if $n \geqq 1$, and $X$ is a point of $P_{n+1}^{\prime}$, then (1) $\phi_{n+1}(X)=\frac{1}{2}$ if $X$ is in $g_{n}^{-1}\left(B_{n}\right),(2)$ $\phi_{n+1}(X)=\frac{1}{2} \phi_{n} g_{n}(X)$ if $X$ is in $\alpha_{n+1}$, and (3) $\phi_{n+1}(X)=1-\frac{1}{2} \phi_{n} g_{n}(X)$ if $X$ is in $\beta_{n+1}$.

By definition, $\phi_{n}\left(A_{n}\right)=0$ and $\phi_{n}\left(B_{n}\right)=1$ if $n=1$; and if it is true when $n=k$, then

$$
\begin{aligned}
& \phi_{k+1}\left(A_{k+1}\right)=\frac{1}{2} \phi_{k} g_{k}\left(A_{k+1}\right)=\frac{1}{2} \phi_{k}\left(A_{k}\right)=0, \quad \text { and } \\
& \phi_{k+1}\left(B_{k+1}\right)=1-\frac{1}{2} \phi_{k} g_{k}\left(B_{k+1}\right)=1-\frac{1}{2} \phi_{k}\left(A_{k}\right)=1 .
\end{aligned}
$$

Also for each $n, \phi_{n} g_{n}=w_{2} \phi_{n+1}$. For suppose $X$ is a point of $P_{n+1}$. If $X$ is in $g_{n}^{-1}\left(B_{n}\right)$, then

$$
\phi_{n} g_{n}(X)=\phi_{n}\left(B_{n}\right)=1=w_{2}\left(\frac{1}{2}\right)=w_{2} \phi_{n+1}(X) .
$$

If $X$ is in $\alpha_{n+1}$, then

$$
w_{2} \phi_{n+1}(X)=2\left[\frac{1}{2} \phi_{n} g_{n}(X)\right]=\phi_{n} g_{n}(X) .
$$

If $X$ is in $\beta_{n+1}$, then

$$
w_{2} \phi_{n+1}(X)=2-2\left[1-\frac{1}{2} \phi_{n} g_{n}(X)\right]=\phi_{n} g_{n}(X) .
$$

Hence the sequence $\left\{\phi_{i}\right\}$ induces a mapping from $\lim \left\{P_{i}, g_{i}\right\}$ onto $D$.

3. Some indecomposable images of $D$. For each $n>1$, let $w_{n}$ denote the map from $I$ onto $I$ such that (1) if $0 \leqq i \leqq n$, then $w_{n}(i / n)$ is 0 if $i$ is even and 1 if $i$ is odd, and (2) $w_{n}$ isl inear on the interval $[i / n,(i+1) / n]$ for each $i(0 \leqq i<n)$. Let $V$ denote the collection of all continua homeomorphic to the limit of an inverse sequence $\left\{I, f_{i}\right\}$ where for each $i, f_{i}=w_{n}$ for some positive integer $n$. The continuum $D$ is an element of $V$ since $D=\lim \left\{I, w_{2}\right\}$.

In this section we show (Theorem 8 ) that $V$ contains every con- 
tinuum homeomorphic to the limit of an inverse sequence $\left\{I, f_{i}\right\}$, where for each $i, f_{i}$ is a nonmonotone near-open map from $I$ onto $I$ (a near-open map is a uniform limit of open maps), and further that each element of $V$ can be mapped onto every element of $V$ (Theorem 9). Hence each element of $V$ is a continuous image of $D$, and therefore of every indecomposable continuum.

It would be of interest to know whether $D$ can be mapped onto every chainable indecomposable continuum with arcwise connected composants. Not every such continuum is an element of $V$ since there is one with three endpoints [7, pp. 141-142], while each element of $V$ has fewer than three. The dyadic solenoid is a nonchainable indecomposable continuum with arcwise connected composants which is not a continuous image of $D[\mathbf{5}]$.

The proof of the following lemma is elementary.

Lemma 1. A map from $I$ onto $I$ is open if and only if there is a sequence $0=a_{0}<a_{1}<\cdots<a_{n}=1$ such that for each positive integer $i, f$ restricted to $\left[a_{i-1}, a_{i}\right]$ is a homeomorphism from $\left[a_{i-1}, a_{i}\right]$ onto $I$.

We call the integer $n$ associated with $f$ in the lemma the order of the open map $f$. Then for each $n, w_{n}$ is an open map from $I$ onto $I$ of order $n$.

Lemma 2. Suppose $\alpha$ is a map from I onto I and $a_{0}, \cdots, a_{n}$ is an increasing sequence of numbers in $I$ such that $\alpha\left(a_{i}\right)=0$ if $i$ is even and $\alpha\left(a_{i}\right)=1$ if $i$ is odd. Then there is a map $\beta$ from $I$ onto $I$ such that $\alpha=w_{n} \beta$. Moreover, if $a_{0}=0, a_{n}=1$, and for each $i, \alpha \mid\left[a_{i-1}, a_{i}\right]$ is a homeomorphism from $\left[a_{i-1}, a_{i}\right]$ onto $I$, then $\beta$ is a homeomorphism from $I$ onto $I$ such that $\beta(0)=0$ and $\beta(1)=1$.

Proof. Let $b_{0}=0, b_{1}=1$ and if $0<i<n$, let $b_{i}=a_{i}$. Let $\beta$ denote the function such that if $b_{i} \leqq x \leqq b_{i+1}$, then $\beta(x)=[i+\alpha(x)] / n$ if $i$ is even, and $\beta(x)=[(i+1)-\alpha(x)] / n$ if $i$ is odd. Clearly $\beta$ is continuous, and straightforward calculations show that (1) $\alpha=w_{n} \beta$, (2) $\beta\left(a_{0}\right)=0$, and (3) $\beta\left(a_{n}\right)=1$. Hence $\beta$ maps $I$ onto $I$.

Definition. If $f$ maps $I$ onto $I$, let $f^{*}=1-f$.

Theorem 5. Suppose $H=\lim \left\{I, f_{i}\right\}$ and $K=\lim \left\{I, g_{i}\right\}$, where for each $i, f_{i}$ is an open map of order $n_{i}$ from $I$ onto $I$, and $g_{i}$ is (1) $w_{n_{i}}$ if $f_{i}(0)=0$, and $(2) w_{n_{i}}^{*}$ if $f_{i}(0)=1$. Then $H$ and $K$ are homeomorphic.

Proof. We define a sequence $\left\{\beta_{i}\right\}$ of homeomorphisms from $I$ onto $I$ such that for each $i, \beta_{i}(0)=0, \beta_{i}(1)=1$, and $\beta_{i} f_{i}=g_{i} \beta_{i+1}$. Let $\beta_{1}$ denote the identity on $I$, and suppose $\beta_{1}, \cdots, \beta_{k}$ have been appropriately defined. Let $0=a_{0}<a_{1}<\cdots<a_{n_{k}}=1$ denote the increasing sequence of numbers associated with the open $\operatorname{map} f_{k}$ as in Lemma 1. 
If $f_{k}(0)=0$, then this sequence satisfies the requirements of Lemma 2, considering $\alpha$ as $\beta_{k} f_{k}$, and there is a homeomorphism $\beta_{k+1}$ from $I$ onto $I$ such that $\beta_{k+1}(0)=0, \beta_{k+1}(1)=1$, and $\beta_{k} f_{k}=w_{n_{k}} \beta_{k+1}=g_{k} \beta_{k+1}$. If $f_{k}(0)=1$, then this sequence satisfies the requirements of Lemma 2, considering $\alpha$ as $1-\beta_{k} f_{k}$, and there is a homeomorphism $\beta_{k+1}$ from $I$ onto $I$ such that $\beta_{k+1}(0)=0, \beta_{k+1}(1)=1$, and $1-\beta_{k} f_{k}=w_{n_{k}} \beta_{k+1}$. Hence $\beta_{k} f_{k}=w_{n_{k}}^{*} \beta_{k+1}=g_{k} \beta_{k+1}$.

The sequence $\left\{\beta_{i}\right\}$ induces a homeomorphism from $H$ onto $K$.

Definition. If $k$ is a positive integer such that if $1 \leqq i \leqq k$, then $n_{i}$ is a positive integer greater than 1 and $g_{i}$ is either $w_{n_{i}}$ or $w_{n_{i}}^{*}$, and $v=g_{1} \circ g_{2} \circ \cdots \circ g_{k}$, then let $|v|$ denote $w_{n_{1}} \circ w_{n_{2}} \circ \cdots \circ w_{n_{k}}$. Let the collection $W$ consist of $w_{2}, w_{3}, \cdots$, and the collection $W^{*}$ consist of $w_{2}, w_{2}^{*}, w_{3}, w_{3}^{*}, \cdots$.

It can be verified that $w_{n} w_{m}=w_{n m}$, so that finite composites of elements of $W$ are in $W$. Furthermore, if $f$ maps $I$ onto $I$, then if $n$ is even, $w_{n} f^{*}=w_{n} f$ and $w_{n}^{*} f=w_{n}^{*} f^{*}=\left(w_{n} f\right)^{*}$, while if $n$ is odd, $w_{n}^{*} f^{*}=w_{n} f$ and $w_{n}^{*} f=w_{n} f^{*}=\left(w_{n} f\right)^{*}$. It follows that if $v_{1}$ and $v_{2}$ are in $W^{*}$, then $v_{1} v_{2}$ is either $\left|v_{1} v_{2}\right|$ or $\left|v_{1} v_{2}\right|^{*}$, so that finite composites of elements of $W^{*}$ are in $W^{*}$.

The next theorem roughly states that any number of maps in an inverse sequence involving $w_{2}, w_{3}, \ldots$ may be "flipped over" without affecting the limit. This is not true in general, for let $f(0)=0, f\left(\frac{1}{2}\right)=1$, $f(1)=\frac{1}{2}$, and $f$ be linear on $\left[0, \frac{1}{2}\right]$ and on $\left[\frac{1}{2}, 1\right]$. Then $\lim \{I, f\}$ is a $\sin (1 / x)$-continuum, while $\lim \left\{I, f^{*}\right\}$ is indecomposable.

THEOREM 6. If, for each $i, n_{i}$ is a positive integer and $g_{i}$ is either $w_{n_{i}}$ or $w_{n_{i}}^{*}$, then $\lim \left\{I, g_{i}\right\}$ and $\lim \left\{I,\left|g_{i}\right|\right\}=\lim \left\{I, w_{n_{i}}\right\}$ are homeomorphic.

Before proving Theorem 6 , we prove a lemma.

LEMma 3. If for each $i, n_{i}$ is an even positive integer, then $H=$ $\lim \left\{I, w_{n_{i}}^{*}\right\}$ and $K=\lim \left\{I, w_{n_{i}}\right\}$ are homeomorphic.

Proof. For each $i$, let $\beta_{i}$ denote the map from $I$ onto $I$ such that $\beta_{i}(x)=1-x$. Then, since $n_{i}$ is even, $\beta_{i} w_{n_{i}}^{*}=w_{n_{i}}=w_{n_{i}} \beta_{i+1}$. Hence the sequence $\left\{\beta_{i}\right\}$ induces a homeomorphism from $H$ on to $K$.

Proof of Theorem 6. We first suppose that for each $i, k_{i}$ is an even positive integer, and that $\left\{j_{i}\right\}$ is an infinite increasing sequence of positive integers such that $g_{j_{i}}=w_{k_{i}}$. Then for each $i$, let $v_{i}=g_{j_{i}+1, j_{i}+1}$. Since $k_{i}$ is even, $w_{k_{i}} v_{i}=w_{k_{i}}\left|v_{i}\right|$ so that $\lim \left\{I, w_{k_{i}} v_{i}\right\}$ is homeomorphic to $\lim \left\{I, w_{k_{i}}\left|v_{i}\right|\right\}$. But the former is homeomorphic to $\lim \left\{I, g_{i}\right\}$, while the latter is homeomoporhic to $\lim \left\{I,\left|g_{i}\right|\right\}$. Under this supposition, then, the theorem is true.

We now suppose that for each $i, k_{i}$ is an even positive integer, and that $\left\{j_{i}\right\}$ is an infinite increasing sequence of positive integers such 
that $g_{j_{i}}=w_{k_{i}}^{*}$. Again for each $i$, let $v_{i}=g_{j_{i}+1, j_{i+1}}$. But $w_{k_{i}}^{*} v_{i}=\left(w_{k_{i}}\left|v_{i}\right|\right)^{*}$, and $w_{k_{i}}\left|v_{i}\right|$ is an element of $W$ of even order. Hence by Lemma 3, $\lim \left\{I, w_{k_{i}}^{*} v_{i}\right\}$ is homeomorphic to $\lim \left\{I, w_{k_{i}}\left|v_{i}\right|\right\}$. But the former is homeomorphic to $\lim \left\{I, g_{i}\right\}$ and the latter to $\lim \left\{I,\left|g_{i}\right|\right\}$.

The only case left is that in which all but finitely many of the integers $\left\{n_{i}\right\}$ are odd, and since for each $n, \lim \left\{I, g_{i}\right\}$ and $\lim \left\{I, g_{i+n}\right\}$ are homeomorphic, we assume that all the integers $\left\{n_{i}\right\}$ are odd. Let $\left\{\beta_{i}\right\}$ denote a sequence of maps from $I$ onto $I$ such that (1) $\beta_{1}$ is the identity on $I$, and (2) $\beta_{j+1}=\beta_{j}$ if $g_{j}=w_{n_{j}}$ and $\beta_{j+1}=\beta_{j}^{*}$ if $g_{j}=w_{n_{j}}^{*}$. Then $\beta_{j} g_{j}=w_{n_{j}} \beta_{j+1}$, and $\left\{\beta_{i}\right\}$ induces a homeomorphism from $\lim \left\{I, g_{i}\right\}$ onto $\lim \left\{I,\left|g_{i}\right|\right\}$.

THEOREm 7. If, for each $i, f_{i}$ is an open map from I onto $I$ of order $n_{i}>1$, then $\lim \left\{I, f_{i}\right\}$ and $\lim \left\{I, w_{n_{\imath}}\right\}$ are homeomorphic.

Proof. This follows directly from Theorems 5 and 6.

THEOREM 8. If, for each $i, f_{i}$ is a nonmonotone near-open map from $I$ onto $I$, then $\lim \left\{I, f_{i}\right\}$ is an element of $V$.

Proof. This follows from Theorem 7 and Brown's approximation theorem [2, Theorem 3, p. 481], since each nonmonotone near-open map is the uniform limit of open maps of order $>1$.

THEOREM 9. If $M$ and $N$ are elements of $V$, then there is a map from $M$ onto $N$.

Proof. $M$ is the limit of an inverse sequence $\left\{I, f_{i}\right\}$ where for each $i, f_{i}$ is in $W$. There is a similar sequence $\left\{I, g_{i}\right\}$ for $N$. For each $i$, let $l_{i}$ denote the order of $g_{i}$. Let $\left\{n_{i}\right\}$ denote an increasing sequence of positive integers such that for each $i$, the order of $f_{n_{i} n_{i+1}}$ is greater than $l_{i}$, and for each $i$, let $f_{i}^{\prime}=f_{n_{i} n_{i+1}}$. Then $\lim \left\{I, f_{i}^{\prime}\right\}$ is homeomorphic to $M$. There is a sequence $\left\{\beta_{i}\right\}$ of maps from I onto $I$ such that $\beta_{1}$ is the identity on $I$ and for each $n, \beta_{n} f_{n}^{\prime}=g_{n} \beta_{n+1}$; for if $\beta_{n}$ is defined, it is easily seen that there is an increasing sequence $a_{0}, \cdots, a_{l_{n}}$ of numbers in $I$ such that $\beta_{n} f_{n}^{\prime}\left(a_{i}\right)$ is 0 if $i$ is even, and 1 if $i$ is odd, and so Lemma 2 gives a map $\beta_{n+1}$ such that $\beta_{n} f_{n}^{\prime}=g_{n} \beta_{n+1}$. The sequence $\left\{\beta_{i}\right\}$ induces a map from $\lim \left\{I, f_{i}^{\prime}\right\}$ onto $N$. So $M$ can be mapped onto $N$.

Corollary. Each element of $V$ is an indecomposable chainable continuum which is a continuous image of every indecomposable continuum.

The referee has suggested that it would be of interest to know whether $V$ contains three topologically different continua. There are two, since $\lim \left\{I, w_{2}\right\}$ has only one endpoint and $\lim \left\{I, w_{3}\right\}$ has two. 


\section{REFERENCES}

1. R. H. Bing, Snake-like continua, Duke Math. J. 18 (1951), 653-663. MR 13, \#265.

2. M. Brown, Some applications of an approximation theorem for inverse limits, Proc. Amer. Math. Soc. 11 (1960), 478-483. MR 22 \#5959.

3. H. Cook, Upper semi-continuous continuum-valued mappings onto circle-like continua, Fund. Math. 60 (1967), 233-239. MR 36 \#3321.

4. L. Fearnley, Characterizations of the continuous images of the pseudoarc, Trans. Amer. Math. Soc. 111 (1964), 380-399. MR 29 \#596.

5. M. K. Fort, Jr., Images of plane continua. Amer. J. Math. 81 (1959), 541-546. MR 21 \#5173.

6. H. Freudenthal, Entwicklungen von Räumen und ihren Gruppen, Composito Math. 4 (1937), 145-234.

7. J. G. Hocking and G. S. Young, Topology, Addison-Wesley, Reading, Mass., 1961. MR 23 \#A2857.

8. W. Hurewicz, Über oberhalb-stetige Zerlegungen von Punktmengen in Kontinua, Fund. Math. 15 (1930), 57-60.

9. A. Lelek, On weakly chainable continua, Fund. Math. 51 (1962/63), 271-282. MR $26 \# 742$.

10. S. Mardesić and J.Segal, $\epsilon$-mappings onto polyhedra, Trans. Amer. Math. Soc. 109 (1963), 146-164. MR 28 \#1592.

11. S. Mazurkiewicz, Sur les images continues des continus, Fund. Math. 17 (1931), 330.

12. J. Mioduszewski, A functional conception of snake-like continua, Fund. Math. 51 (1962/63), 179-189. MR 26 \#1859.

13. J. T. Rogers, Jr., Pseudo-circles and universal circularly chainable continua, Doctoral Dissertation, University of California, Riverside, Calif., 1968.

Emory University, Atlanta, Georgia 30322 